\title{
Protocolo de clase como actividad orientadora de enseñanza en el grado segundo de Primaria
}

\author{
Claudia Patricia Perlaza Díaz ${ }^{*}$ \\ Verónica María Henao Ramírez ${ }^{* *}$ \\ Recibido: 15 de febrero de 2017 \\ Enviado a pares evaluadores: 20 de febrero de 2017 \\ Aprobado por pares evaluadores: 22 de marzo de 2017 \\ Aprobado por comité editorial: 17 de mayo de 2017 \\ DOI: 10.22395/csye.v6n11a4
}

\section{RESUMEN}

Este artículo da cuenta del proceso, los hallazgos y los resultados de una práctica investigativa en la Institución Educativa José Félix de Restrepo Vélez, específicamente en el grado segundo de primaria, cuyos estudiantes viven en el municipio de Sabaneta donde se encuentra ubicado el plantel.

La modalidad de la investigación fue de tipo cualitativa, porque desde el diseño etnográfico aplicamos la técnica de observación participante, e implementamos como instrumento el protocolo de clase como actividad orientadora de enseñanza, desde el cual se desarrollaron las unidades didácticas centradas en el aprendizaje de los conceptos de suma y resta, aplicados en la solución de problemas matemáticos.
Los registros y datos fueron las producciones de los estudiantes, y su análisis se hizo tomando como referencia principal la perspectiva histórico-cultural, ya que complementada con otros postulados teóricos, nos permitió comprender aspectos importantes sobre la enseñanza y el aprendizaje de las matemáticas.

Los resultados obtenidos a partir de esta experiencia evidencian cómo la estructuración de nuestra actividad docente posibilitó a los estudiantes dar un salto cognitivo (Vigotsky, 1983) en el aprendizaje de los conceptos de suma y resta aplicados en la solución de problemas matemáticos.

Palabras clave: perspectiva históricocultural, formación de conceptos matemáticos y actividades orientadoras de enseñanza.

Magíster en Educación de la Universidad de Antioquia y Licenciada en Educación Especial Infantil de la misma universidad. Docente de Básica Primaria de la Institución Educativa José Félix de Restrepo Vélez del municipio de Sabaneta. Correo electrónico: clapapedi@gmail.com

** Magíster en Educación de la Universidad de Antioquia e ingeniera Electricista de la misma universidad. Docente de Básica Primaria de la Institución Educativa José Félix de Restrepo Vélez del municipio de Sabaneta. Correo electrónico: veroh79@msn.com 


\section{Class protocol as a guiding activity in teaching at the second grade of elementary school}

\section{ABSTRACT}

This investigative proposal intends to give an account of the process, the findings and results that emerged during our practice as research teachers, which was held in the educational institution José Félix de Restrepo Vélez, specifically in second grade, whose students live in the municipality of Sabaneta where the campus is located.

The research is qualitative; from the ethnographic design type, we applied the technical participant observation and we implemented as tool, the class protocol as their guiding teaching room which the didactic units focused on learning the concepts of addition and subtraction were developed, applied in the solution of mathematical problems.
Records and data analyzed were the productions of the students and their analysis was performed taking as main reference the perspective historic-cultural since supplemented by other theorists postulated allowed us to understand important aspects of the mathematics teaching and learning.

The results obtained from this experience allowed us to demonstrate how the structuring of our teaching allowed students to take a cognitive leap (Vigotsky, 1983), in learning the concepts of addition and subtraction applied in solving mathematical problems.

Keywords: perspective historic-cultural, concept mathematician formation and their guiding teaching room. 


\section{Introducción}

La propuesta pedagógica que se presenta a continuación mejoró la comprensión y la apropiación de los conceptos matemáticos; a su vez, desde la revisión bibliográfica y la práctica se realiza un aporte significativo a la Institución Educativa José Félix de Restrepo Vélez del municipio de Sabaneta. Frente a las dificultades que tenían los estudiantes para apropiarse de manera adecuada de los conceptos matemáticos de suma y resta, se planteó como eje central diseñar y aplicar un protocolo de clase como actividad orientadora de enseñanza. Para ello se evaluó la efectividad de la propuesta durante el primero y segundo períodos académicos del calendario escolar de 2016.

El marco teórico parte desde la mirada del Ministerio de Educación Nacional (MEN) sobre los procesos formativos para el área de matemáticas, seguido de los aportes de la perspectiva histórico-cultural frente a la actividad orientadora de enseñanza; por último, se abordan aspectos como la formación de conceptos y la importancia de la solución de problemas matemáticos en la Básica Primaria. Esta investigación se desarrolla desde la modalidad cualitativa, metodología tipo etnográfico.

Adicionalmente, se describen los diferentes pasos del protocolo de clase y se presentan los resultados y las conclusiones que surgieron de la misma, a través de las cuales se da respuesta a la pregunta de investigación.

\section{Hopizonte teórico (antecedentes)}

La perspectiva histórico-cultural desde Vigotsky (1983) se construyó en la lucha contra dos frentes de la psicología del momento: el primero, que biologizaba la conducta y el pensamiento, y el segundola, que trataba las funciones psíquicas como producto de un psiquismo autónomo, abstraído del medio. Su método fue el histórico-genético que recoge la evolución filogenética y ontogenética, pero en una determinación histórico-cultural del hombre. Esto le permitió realizar diferentes aportes para la enseñanza y la educación, de los cuales, decidimos retomar el concepto de actividad para ampliar nuestra investigación. Este término concibe la actividad humana como objetiva, en oposición a los que ven la realidad y la sensoriedad bajo la forma de objeto o de contemplación. Desde esta mirada, la actividad es considerada como el elemento central de la perspectiva histórico-cultural.

Marx, en su tesis sobre Feuerbach, considera como un principio de la actuación la "práctica-crítica", es decir, el pensamiento humano ligado a la práctica, ya que es mediante esta donde se confirma si las ideas corresponden con el mundo exterior (Marx, 1845). 
Según la postura histórico-cultural, la actividad fundamental del hombre es el trabajo; mediante este el hombre vuelve la mirada a sí mismo, y utiliza tanto los instrumentos como el lenguaje para mediar su realidad. En el proceso de transformación del mono en hombre estas actividades se vuelven cultura, y una de esas actividades es la relacionada con el acto de enseñar y el acto de aprender en un contexto social como la escuela, donde el estudiante desarrolla su personalidad.

Aunque los seres humanos son resultado de los entornos y de la educación, también son el producto de circunstancias distintas que, a su vez, pueden cambiarse o transformase: los docentes pueden educar y ser educados. Por tanto, la actividad así se convierte en un objeto, y este objeto debe ser pensado desde las prácticas docentes para formar conceptos en la mente humana que generen un conocimiento.

Al estudiar diversas teorías se encontró que el MEN, a través de los lineamientos curriculares, genera interrogantes como: ¿qué son las matemáticas?, ¿en qué consiste la actividad matemática en la escuela?, ¿para qué y cómo se enseñan las matemáticas?, ¿qué relación se establece entre las matemáticas y la cultura?, ¿cómo se puede organizar el currículo de matemáticas?, ¿qué énfasis es necesario hacer?, ¿qué principios, estrategias y criterios orientarían la evaluación del desempeño matemático de los estudiantes? (p. 9). La búsqueda de respuestas a estas reflexiones permitió hacer una aproximación a posiciones gnoseológicas y metodológicas que responden de una manera específica a dichos interrogantes.

En este orden de ideas, se encuentran autores como Carolina Douglas, Guillermo Bernaza y Roberto Corral (2006), quienes en su artículo "Una propuesta didáctica para el aprendizaje de la física", apoyados en los postulados de Vigotsky, proponen, para organizar la enseñanza de conceptos en esta área, los siguientes pasos: a) comprender que los estudiantes poseen unos preconceptos sobre los fenómenos que analizan, b) partir desde sus propias experiencias, c) tener en cuenta su nivel lingüístico, d) presentar el lenguaje simbólico de la física, e) propiciarles la expresión verbal y escrita, f) aclarar y complementar el correlato que presentan los educandos y g) permitir que el trabajo consciente e intencional esté en función de los objetivos propuestos con la ayuda de medios materiales. Para los autores es de suma importancia la discusión sobre la solución de problemas físicos, ya que esto propicia que el conocimiento sea adquirido y reforzado desde la sana discusión de las formas de solucionarlos; también sugieren que en este proceso se lleve al estudiante a que describa con sus palabras lo que ocurre, analice los datos e identifique a qué ley física se refiere el problema; acto seguido, que represente la situación con una gráfica, aplique los modelos necesarios para la solución y pueda analizar los resultados. 
Durante la práctica pedagógica es fundamental considerar que la solución de problemas matemáticos es una actividad que potencia el desarrollo de los procesos psicológicos superiores, pero esta actividad requiere ser organizada y mediada por los docentes para llevar a los estudiantes de su zona de desarrollo real a una zona de desarrollo próximo. En este sentido, cobra importancia la actividad mediadora del docente; de ahí que la propuesta metodológica de George Polya, citado por la Isabel Echenique, resulte llamativa cuando propone cuatro etapas a seguir para la solución de problemas matemáticos: comprender el problema, concebir un plan, ejecutar el plan y devolvernos sobre lo ejecutado (visión retrospectiva); al aplicar cada uno de estos pasos se pueden identificar las dificultades particulares de cada estudiante, para poder mediarlas a través de las actividades orientadoras de enseñanza. Esta autora también plantea una tipología de problemas a trabajar en la Básica Primaria, entre los cuales, y para efectos de esta investigación solo se retomaron los problemas aritméticos de primer nivel o de un solo paso, ya que se requiere de una sola operación para su resolución, los cuales clasifican de la siguiente manera: de cambio, de combinación, de comparación y de igualación, utilizados para la enseñanza de la adición y la sustracción en el primer ciclo de Básica Primaria.

\section{Marco teórico}

\section{Formación de conceptos}

Con el avance científico han surgido teorías, tales como aproximaciones innatistas, asociacionistas, del conocimiento nuclear y socio-cultural, que han centrado su interés en investigar las fuentes de información y procesos que dan cuenta de la formación de conceptos, a través de la comprensión de los significados posibles de las palabras. Dichas propuestas teóricas concuerdan en que los conceptos cumplen un rol importante en el desarrollo cognitivo, pero difieren marcadamente en el modo de explicar su adquisición y desarrollo temprano. Las teorías individualistas (la innatista, la asociacionista y la nuclear) se desarrollan en relación con la naturaleza (entendida como el objeto) y la experiencia del sujeto mediante la enseñanza por el descubrimiento. También se suman las teorías de tipo desarrollista que se inclinan más por las corrientes constructivistas, y definen la formación de conceptos como un proceso individual, que se da por etapas o estadios de desarrollo, mediante la enseñanza por el descubrimiento, como lo enuncia Piaget.

A diferencia de las anteriores, las teorías socio-culturales ponen mayor acento en los procesos que permiten la formación y desarrollo del significado, que se desenvuelven inicialmente en el plano intersubjetivo, para luego interiorizarse en el plano intrasubjetivo (Sabina y Peralta, 2008). 
Otra opinión es la de Vigotsky, quien le da mucha importancia a la construcción del conocimiento mediante las interacciones sociales, ya que estas son pilares para el surgimiento del pensamiento. De ahí que sea importante el contexto en el que se desenvuelve el niño, el cual posibilita nuevos intercambios culturales que le estimulen el intelecto y le creen nuevas finalidades para alcanzar estadios superiores del pensamiento. Estos postulados se podrían complementar con los aportes teóricos de Ausubel, quien considera fundamental la estructura cognoscitiva que tiene cada sujeto para la construcción del conocimiento y la interacción activa que se da con los conocimientos previos, los cuales se pueden evidenciar cuando un estudiante adquiere un aprendizaje significativo, al incorporar lo que está percibiendo, ya sea un objeto, una cosa, un animal, entre otros, y que, a su vez, representa el concepto que va adquiriendo durante una experiencia de aprendizaje,es decir, cuando logra representar por medio de la palabra hablada o escrita el objeto o realidad a los cuales se refiere (Delgado, Arrieta y Campo, 2012).

A continuación, se cita como ejemplo una situación que se vivió en aula de clase, cuando una estudiante pregunta qué es curiosidad, y sucesivamente cada compañero le va dando su definición por medio de ejemplos, sumado el aporte de la docente, hasta que la niña establece una relación entre dicha información con sus saberes previos y logra relacionar las expresiones y definiciones de sus compañeros con sus propias experiencias de aprendizaje, evocando y nombrando momentos de su vida cuando se interesó en algo y quiso saber sobre eso. Se puede evidenciar que la estudiante escucha una palabra desconocida, y luego de comprender su significado siente la necesidad de usarla en sus conversaciones, haciendo de esta un elemento más de su lenguaje cotidiano. En este momento del aprendizaje es importante el rol del docente, quien debe tener en cuenta las diferentes etapas en la adquisición de conceptos, cooperando de manera sistemática con el estudiante, al explicarle y brindarle información, llevándolo a que se cuestione y modifique su concepto, si es necesario, hasta lograr que este verbalice sus pensamientos. Esta situación también nos lleva a recordar que "una palabra sin significado es un sonido vacío; el significado es, por lo tanto, un criterio de la palabra y su componente indispensable" (Vigotsky, 1983, p. 26).

El Ministerio de Educación Nacional (MEN) colombiano, desde los lineamientos curriculares, realiza una propuesta que se podría relacionar con la teoría de Vigotsky, cuando reconoce: "Que el conocimiento matemático es resultado de una evolución histórica, de un proceso cultural" (p. 14), y se valora "la importancia que tienen los procesos constructivos y de interacción social en la enseñanza y en el aprendizaje de las matemáticas" (p. 14); a su vez, habla sobre la significación en la caracterización de la función simbólica como un contacto que se da entre la mente del sujeto y la cultura, llevándolo a crear nuevos significados del mundo 
que construye, y desde los estándares se centran la atención y el interés en el fortalecimiento de las habilidades del lenguaje que va adquiriendo.

A partir de los anteriores postulados teóricos, podríamos concluir que el conceptoes la parte esencial de las palabras que facilitan los procesos de comunicación y que ayudan a dar significado a la realidad. La conceptualización matemática y la comprensión de lectura de los textos matemáticos son esenciales en el proceso de aprendizaje del estudiante, quien desde las distintas áreas del conocimiento se ve enfrentado a diferentes tipos de conceptos durante su tránsito por la escuela, para poder transmitir en forma verbal y escrita lo aprendido; por ello, no tener estas herramientas puede afectar su desempeño académico, en la medida en que no logra identificar el sentido del concepto en el texto y, a su vez, afecta la relación con el otro y su motivación e interés hacia el aprendizaje.

\section{Conceptos matemáticos}

Durante la enseñanza de las matemáticas en edad preescolar, se tiende a pensar que el hecho de que los niños reciten los números de manera mecánica implica que ellos ya sepan contar, sin considerar que son simplemente sonidos carentes de significado, ya que la creación del concepto de número no se ha generado aún y, por lo tanto, no se ha dado una comprensión real del significado de dichos conceptos (Wallon, 1979 y Kamii, 1986, citados por Frontera, 1992, p. 33).

Continuando con las apreciaciones de la autora, sobre este aspecto, Piaget opina que la adquisición de conceptos matemáticos debe darse de manera ordenada, y requiere de procesos anteriores, por ejemplo: para entender el concepto de número, adición y sustracción se deben tener los conceptos previos de seriación, clasificación, correspondencia y conservación, aspectos que aparecen desde la etapa operacional concreta (Frontera, 1992, p. 34). El estudiante, al llegar a niveles mayores de escolarización, hace un acto consciente y reflexivo sobre lo que está aprendiendo y descifra el significado real de los conceptos que ha estado utilizando, pasando del acto de enunciar los números a la comprensión real y la apropiación del concepto; por ejemplo, el número "3" para ellos representa una posición antes que una cantidad compuesta por tres unidades y que esta se puede descomponer también en un grupo de una unidad más dos unidades (Frontera, 1992). Por lo tanto, los conceptos matemáticos y los procedimientos (el desarrollo de técnicas y conceptos) que intervienen conjuntamente cuando los niños aprenden a contar están entrelazados. Los conceptos numéricos y contar significativamente se desarrollan de forma gradual, dando como resultado la aplicación de técnicas para contar más complejas, y conceptos cada vez más elaborados (Frontera, 1992, p. 42). Como es bien sabido, comprender es aprender el significado. El significado es una idea. Las ideas son elementos constitutivos 
imprescindibles. Aprender, pues, el significado de una cosa, un acontecimiento, o una situación es contemplarlo en sus relaciones con otras cosas, observar cómo opera o cómo funciona, qué consecuencias se siguen de él, qué lo produce, qué utilidad puede dársele (Dewey, 1993, p. 121).

Cuando un estudiante debe comprender un texto en alguna asignatura, de antemano debe conocer los términos que en ella se manejan; es como conocer las reglas del juego antes de comenzar a jugar. Para esto debe contar con el respaldo del docente, quien es el que debe tener mayor capacidad de enseñar a leer los códigos y cómo operar con ellos, sobre todo en el áreas de matemáticas, ya que no es lo mismo leer textos en literatura que textos especializados; no solo se trata de mecanizar ejercicios sino también de comprender los enunciados que allí se presentan, saber cuál es el significado de cada palabra, extrayendo la información necesaria, y tener clara la operación que debe realizar para llegar a un resultado favorable (Jurado, 2004).

Autores como Miguel de Zubiría y Julián de Zubiría (1992), en el libro Biografía del pensamiento, estrategias para el desarrollo de la inteligencia, hacen hincapié en que la educación matemática en Primaria es aprender las leyes básicas, es decir, asimilar y aprender los conceptos de sistemas y subclasificación de los números naturales, operaciones (sucesor, antecesor, suma, resta, multiplicación, división y radicación), establecimiento de relaciones (igualdad, mayor que, menor que), equivalencia, simetría, pertenencia e inclusión. Dado que los estudiantes del grupo experimental se encuentran en el primer ciclo de Educación Primaria, para efectos de esta investigación se hizo énfasis en conceptos como valor posicional, suma y resta como la puerta de entrada para la solución de problemas aritméticos, conceptos que son una aplicación directa del pensamiento matemático en la vida real del estudiante.

\section{Problemas matemáticos}

Según Ernesto García Alzola (1975), el sentido de los textos no está determinado por el significado propio de cada palabra, sino por la relación que se establece entre ellas, con la ayuda de los signos auxiliares (juntivos, signos de puntuación, marcadores textuales). Además, para una buena lectura no basta con entender el significado de cada palabra y de las relaciones que se establecen entre ellas, sino que es necesario ubicar estos sentidos en los contextos sociales y culturales en que fueron producidos y desde donde se leen (Castañeda y Henao, 1995, p. 8).

En la solución de problemas matemáticos, una de las dificultades presentadas es la comprensión de los conceptos por parte de los estudiantes, y puede deberse a los significados que presentan las palabras, que en la vida cotidiana significan algo ,y dentro de los problemas su significado es diferente; por ejemplo, 
cuando se hace uso de la palabra diferencia (para enunciar una resta) esta puede tomar diversos significados para los estudiantes, tales como: si los números presentados son pares o impares; si son primos o no; cuántos números existen entre ellos, es decir, que no son iguales como lo expresan nuestros estudiantes. Otra dificultad para la comprensión de problemas matemáticos radica en que se pueden presentar diversas formas de formular una misma pregunta, lo que puede generar confusión afectando la transcripción del enunciado al lenguaje matemático, además de la brecha existente entre los problemas presentados en la escuela y en la vida real. Esto se muestra cuando un niño va a la tienda de compras y realiza las operaciones como son debidas, cuánto tiene, para qué le alcanza y cuánto le deben devolver, pero si en clase se le enuncia un problema parecido a este dice que no es capaz o que no lo entiende. Por esta razón, los problemas presentados en clase deben ser modificados y se deben acercar más a la cotidianidad, lo que logrará que los estudiantes alcancen una competencia matemática real (Blanco y Blanco, 2009).

Como lo describe Manuel Santos Trigo (1997) en su escrito Principios y métodos de la resolución de problemas en el aprendizaje de las matemáticas, la solución de problemas es de suma importancia en el desarrollo mental de los estudiantes, y existen varias formas de incrementar dicho desarrollo, como cita el matemático Schoenfeld, quien dice que es importante que el docente, por lo menos una vez a la semana, resuelva ejercicios en el tablero para que los estudiantes observen cómo se enfrenta a la solución de problemas y las estrategias que utiliza para esto; también deben observar a sus compañeros resolver problemas, discutir sobre las destrezas o falencias que poseen para hacerlo, narrarles sus estrategias de solución, por qué eligen un método en particular y para qué les sirve cada operación que realiza. También se les deben plantear problemas que tengan diversos métodos de solución para que ellos entiendan que en ocasiones no existe un solo método y que deben buscar el mejor; además, que diseñen o replanteen problemas para afianzar más su conocimiento. Para Schoenfeld, citado por Santos, la solución de problemas tiene varias fases importantes: el análisis (dibujar el problema si es necesario, simplificarlo y hallarle el sentido), la exploración (renombrar los elementos, remplazar condiciones por equivalentes, reformular el problema, entre otros) y la verificación de la solución (uso de datos pertinentes, que concuerde la respuesta con sus predicciones y analizar si existen otras formas de solucionarlo). Haciendo un buen uso de estas fases los estudiantes se podrán sentir seguros de su proceso y en capacidad de convencer a sus compañeros sobre la forma de realizarlo, mostrando que su conocimiento matemático es adecuado.

En la actualidad, la mayoría de los métodos de solución se encuentran basados en los aportes de Polya, que consisten en cuatro pasos esenciales cuyo 
desarrollo se deben presentar en un clima idóneo, que los estudiantes estén motivados y sin afanes:

1. Comprender el problema: implica no solo comprender el texto y todos los conceptos allí expresados, sino también la situación problema que se presenta. Es decir, descodificar el mensaje y llevarlo al lenguaje matemático.

2. Hacer un plan para resolver el problema: es de suma importancia en esta fase identificar los datos que se presentan en el problema, qué operación se debe utilizar y el orden que se debe llevar para solucionarlo.

3. Poner el plan en práctica: es realizar cada uno de los pasos que se presentan en la fase anterior y que llevan directamente a encontrar una solución al problema.

4. Examinar lo realizado: hallar la respuesta de un problema matemático no es la finalización de la actividad; esta es la parte importante, ya que el estudiante debe analizar si la respuesta que encontró es pertinente a la pregunta que se estableció en el problema, reflexionar si esta forma de solucionar resulta eficiente o si se puede establecer una más corta, y si es el caso, esta forma de solucionar se puede aplicar a otras situaciones. También es necesario que los estudiantes logren verbalizar la estrategia que utilizaron para solucionar el problema; de esta manera se puede conocer su forma de razonar e identificar si existen lagunas en el conocimiento del estudiante (Echenique, 2006).

Para concienciar al estudiante de estos procesos, en un principio se le formulan las siguientes preguntas:

1. ¿Qué dice el problema?

2. ¿Cuáles son los datos del problema?

3. ¿Qué operación se debe realizar?

4. Cuadro de operaciones.

A medida que el estudiante interioriza estos pasos va a resolver problemas matemáticos de una forma más rápida y eficaz (Barcena et al., 2009).

\section{Actividad orientadora de enseñanza}

El aprendizaje de los conceptos matemáticos debe ir acompañado de la idoneidad didáctica, que consta de varios apartados: idoneidad epistémica, es decir, 
problemas acordes con el contexto y el nivel de dificultad adecuados; idoneidad cognitiva, esto es, los temas deben concordar con el desarrollo cognitivo y con los conocimientos previos del estudiante y, además, deben ser alcanzables; idoneidad interaccional, que la explicación del docente debe ser clara y favorecer el diálogo con los estudiantes; idoneidad mediacional, es decir, tener los recursos y materiales para una buena clase y que el tiempo sea invertido en los conceptos nucleares; idoneidad afectiva, que se tenga interés y motivación por parte del estudiante, e idoneidad ecológica, que los conocimientos se encuentren ligados al PEI y al contexto del estudiante (Godino y Neto, 2013).

De la mano con la idoneidad debe ir la organización propia de la enseñanza que hace el profesor; en este sentido, retomamos los lineamientos curriculares del MEN 1998, en los cuales se considera que:

El conocimiento matemático es considerado hoy día como una actividad social que debe tener en cuenta los intereses y la afectividad de los estudiantes... Su valor principal está en que organiza y da sentido a una serie de prácticas, a cuyo dominio hay que dar esfuerzo individual y colectivo. La tarea del educador matemático conlleva entonces una gran responsabilidad, puesto que las matemáticas son una herramienta intelectual (p. 14).

Desde estos postulados, se plantea el protocolo de clase-Actividad orientadora de enseñanza- como una alternativa para organizar los procesos de enseñanza, en este caso, los conceptos de suma y resta para ser aplicados en la solución de problemas matemáticos, y principalmente, como la posibilidad de contribuir en el desarrollo de las actividades de aprendizaje de los estudiantes referidos a la apropiación de algunos conceptos matemáticos, para que mejoren el bajo desempeño en esta área.

\section{Definición de protocolo}

Se podría definir el protocolo como el conjunto de normas o procedimientos que explican lo que se debe hacer, según el caso, o como la organización de actos sociales y sus normas de etiqueta y el tratamiento que se les da a los símbolos patrios. Pero esta palabra es también usada en la empresa, en la etiqueta, en la cortesía, en el vestido, entre otros (Solé, 2006).

La palabra protocolo es también utilizada en el área de la medicina y es definida como los documentos que ayudan al personal médico para prestar una atención de calidad permitiendo tomar decisiones en momentos de incertidumbre; estos también contribuyen a normalizar la práctica, facilitando a las personas que ingresan nuevas a las instituciones realizar su labor de la mejor manera. Este protocolo es un acuerdo entre los expertos del área para clarificar las ideas a aplicar ante una determinada tarea. Estos documentos deben ser 
revisados periódicamente y modificados, si es el caso. No existe aún un orden específico para su creación, pero sí se deben tener en cuenta algunos aspectos relevantes como las personas que lo crearon, quiénes lo deben utilizar y en qué momento, fecha de elaboración y revisión, objetivos del protocolo y el material que se debe utilizar (Sánchez et al. 2009).

Otro término que se conoce es el protocolo académico, y este hace referencia a una síntesis que ordena los puntos más importantes tratados en un evento académico, el cual contiene una descripción objetiva de los temas tratados, los hechos o sucesos del evento, un texto escrito de los aspectos más fundamentales del trabajo realizado, y no es un acta ni un simple resumen del evento; tampoco es un ensayo o una recopilación de opiniones sueltas. El protocolo, como tal, cuenta con unas propiedades, las cuales son: colocar las opiniones sueltas como ideas con sentido, que las personas ausentes puedan enterarse del trabajo realizado en la sesión, tener la atención de los participantes en los temas centrales tratados, reforzar el proceso de enseñanza-aprendizaje fijando los campos de la información, contar con una descripción narrada que facilita la construcción de las memorias e identificar los temas en los cuales el grupo tuvo una adecuada o deficiente asimilación de los contenidos. Su estructura debe contar con la descripción del lugar, hora, participantes, motivo del evento, duración, descripción de las actividades, temas tratados, decisiones o acuerdos fundamentales y tareas asignadas. Cuando se construye un protocolo las personas deben saber distinguir entre los comentarios sueltos y los reales aportes al tema, ya que los primeros pueden llegar a confundir a los que luego abordan el documento para enterarse de lo sucedido o recordarlo. Este debe ser leído en el grupo de trabajo y de esta manera concretar cuáles son los temas verdaderamente importantes y complementarlos, según sea el caso y, además, eliminar los que no lo son para el grupo (Rengifo, 2010).

Para el equipo investigador, el protocolo de clase es un documento construido con unos pasos esenciales, según el soporte teórico de la investigación, que propicia la adquisición de conceptos en los estudiantes, no solo en el área de matemática sino desde cualquier área del conocimiento. Este fue construido con base en la indagación de varios autores como Vigotsky, Frontera (1992), Dewey (1993), Jurado (2004), Echenique(2006), los hermanos De Zubiria (1992), quienes presentan diversas estrategias a considerar sobre el orden que se podría llevar cuando se enseña un concepto. De esta manera se estructura el formato de planeación de clase, donde se plantean los diferentes pasos del protocolo que le permitan al estudiante la comprensión y apropiación de los nuevos conceptos. Además, ayuda al docente en la organización y ejecución de su clase. 


\section{Metodología}

La investigación de tipo cualitativo es inductiva, holística, interactiva y reflexiva, abierta y humanista (Tamayo, 1999). Desde ella el investigador debe tener una relación cercana, empática y con una buena comunicación con el objeto de estudio, logrando interactuar con los individuos en su contexto social, con la intención de explorar, describir y comprender dicha realidad, partiendo de un proceso de interpretación y construcción teórica que posteriormente habrá de plasmar en el proyecto de investigación.

El diseño implementado fue la etnografía, ya que estudia los fenómenos sociales que son fruto de la interacción de un grupo incluyendo el escolar, y permite el desarrollo de los procesos de construcción teórica. Según Fetterman, citado por Hernández Sampieri: "La educación etnográfica puede comenzar con un modelo o teoría, o bien directamente con el trabajo de campo e ir agregando la revisión de la literatura" (2015, p. 484).

Una característica fundamental de este diseño es que ofrece instrumentos de investigación como la observación participante de la práctica pedagógica, la cual fue sistematizada mediante el diario de campo y el portafolio de evidencias (documentos); ambos arrojaron datos que fueron analizados para sacar las conclusiones pertinentes a la investigación (Martínez, 2011).

La construcción del formato y del instrumento para la evaluación diagnóstica, el pretest y el postest surgen de la propuesta de Juan Carlos Negret Paredes (2008), dado que aporta elementos fundamentales desde el eje de pensamiento lógico-matemático al pretender que los estudiantes aprendan a operar con números de manera oral y escrita, y puedan pasar de las operaciones básicas concretas a las operaciones aritméticas formales con números y algoritmos escritos, requeridos para el desarrollo de competencias aritméticas básicas. También ofrece una alternativa para la educación flexible al permitir la adquisición de la habilidades y competencias de lectura, escritura y lógico-matemáticas a través de la metodología de proyectos de aula, logrando con ello el acceso de los niños al aula regular y el reconocimiento de sus derechos esenciales para la inclusión social y educativa (2008). En este sentido, se logra que el estudiante se motive, sea el protagonista de su propia educación y acceda a los planes curriculares de la asignatura y se generen aprendizajes significativos.

\section{Protagonistas de la investigación}

La perspectiva histórico-cultural plantea el desarrollo cognitivo desde una visión social, y de acuerdo al Modelo Pedagógico de la institución José Félix de Restrepo Vélez: "El enfoque inclusivo se basa en la valoración de la diversi- 
dad como elemento enriquecedor del proceso de enseñanza-aprendizaje y en consecuencia favorecedor del desarrollo humano" (p. 24); de ahí que el grupo experimental está conformado por estudiantes de segundo de Primaria, cuyas edades oscilan entre 6 y 9 años, de los cuales 9 presentan algún tipo de diagnóstico de tipo cognitivo, psicosocial (déficit de atención con y sin hiperactividad) o un trastorno de lenguaje.

Las intervenciones pedagógicas se desarrollaron en la Institución Educativa José Félix de Restrepo Vélez en el municipio de Sabaneta, durante la jornada escolar habitual de los estudiantes, en la intensidad horaria estipulada para el área de matemáticas, definida por el Consejo Académico de la institución.

Los criterios que se tuvieron en cuenta para la selección de los participantes fueron los siguientes:

- Que fueran estudiantes de la institución educativa, y pertenecieran a los grupos donde las investigadoras realizaban sus actividades pedagógicas.

- Se seleccionó el grado segundo de Básica Primaria porque hubo disposición y el tiempo (jornada de clase) para ejecutar el proyecto de investigación.

- Se contó tanto con la autorización de los acudientes y padres de familia de los estudiantes, así como de las directivas de la institución para que las producciones (orales y escritas) y grabaciones pudieran ser utilizadas, estudiadas y publicadas con fines académicos, bajo códigos, protegiendo siempre la identidad y privacidad de los estudiantes.

- Los principios y valores instituciones contemplados en el PEI y su mirada sobre la inclusión educativa garantizan el derecho a la participación social de las personas, dado que sus condiciones de discapacidad, etnia, religión o sexo no pueden ser motivo de exclusión.

\section{Trabajo de campo}

El trabajo de campo del presente proyecto se inició durante el primer semestre del año 2016, correspondiente al primero y segundo períodos académicos de la institución. Se realizaron 12 sesiones de clase con el grupo experimental (30 estudiantes) y dos sesiones con un grupo control (29 estudiantes). Cada sesión tuvo una duración aproximada de 2 horas distribuidas de la siguiente manera: 20 minutos de actividades de rutina (saludo, registro de asistencia, realización de la agenda y pactos de convivencia) y 1 hora 40 minutos para el desarrollo de la clase, acorde con el protocolo propuesto para la investigación. El desarrollo metodológico se realizó en cuatro momentos, distribuidos así: 
Momento 1. Revisión de literatura antecedente, los lineamientos curriculares del área de matemáticas estipulados por el MEN, los estándares básicos de competencias matemáticas, la malla curricular municipal y los derechos básicos de aprendizaje de matemáticas con el fin de replantear y / o diseñar los indicadores de desempeño a partir de los cuales se realizarían las planeaciones para el primero y segundo períodos académicos de año 2016.

Momento 2. Selección, elaboración de formatos a implementar para la planeación, la sistematización del diario de campo y de los instrumentos de evaluación diagnóstica.

Momento 3. Aplicación del protocolo de la clase como actividad orientadora de la enseñanza en las diferentes sesiones o clases.

Momento 4. Sistematización y análisis de la actividad de aprendizaje y de enseñanza.

\section{Análisis de datos}

Los resultados del análisis se obtienen de la triangulación entre las producciones (pruebas y talleres realizados a los 30 estudiantes) y las gráficas donde se establece la diferencia entre los resultados de los estudiantes con necesidades educativas especiales (NEE), sin necesidades educativas especiales, y el grupo control (que solo se tuvo en cuenta como elemento comparativo para el postest). Se comparan los resultados con el fin de poder dar respuesta a la pregunta de investigación determinando si los estudiantes obtienen, o no, mejores resultados con la implementación del protocolo de clase como actividad orientadora de la enseñanza, a la vez que se verifica la efectividad del protocolo mismo.

\section{Resultados del test inicial o evaluación diagnóstica: pensamiento numérico y sistemas numéricos}

Este instrumento permitió conocer el estado inicial de los estudiantes frente a la apropiación del concepto de número en general, y evidenciar que reconocen y manejan los signos que representa cada uno de los números dígitos, comparan las cualidades particulares de los conjuntos, establecen relaciones de cantidad, correspondencia, orden y secuencia haciendo uso correcto del signo escrito, realizan conteo (progresivo y regresivo), logran componer y descomponer cantidades usando la base del 10. También se evidenció que los educandos presentaban mayor dificultad en los componentes relacionados con la comprensión del valor posicional (capacidad de hacer corresponder a cada número dentro de una cifra, la cantidad de elementos que representa según su valor posicional), las situaciones aditivas y de sustracciones numéricas simples (en relación con la capacidad para formular, analizar y resolver situaciones matemáticas que involucren estas 
operaciones) y las operaciones de suma o resta por complemento; principalmente resaltaron dificultades para aplicar estos conceptos en la solución de problemas matemáticos. De ahí que la investigación se haya centrado en estos últimos tres aspectos y se establecieran diferentes criterios de evaluación para cada uno de ellos, clasificados en los niveles de insuficiente, básico, superior y excelente correspondientes al sistema de evaluación de la institución educativa.

\section{Resultados del pretest y el postest según las diferentes unidades de análisis}

\section{Valor posicional}

Los resultados correspondientes a los estudiantes sin NEE permiten deducir que el grupo experimental $2 .^{\circ}$ evidencia avances significativos, dado que la generalidad, $85.7 \%$ de los estudiantes, se ubica en el nivel $\mathrm{E}$, es decir, que logran reconocer y representar el valor posicional solamente de unidades de primero, segundo y tercer orden, sin presentar ningún error. Los estudiantes del grupo control están ubicados en los cuatro niveles haciendo de este un grupo heterogéneo, y solo el $48 \%$ se encuentra en el nivel E, frente a la ubicación de las cantidades según su valor, lo cual requiere que el docente en su práctica utilice estrategias diferenciadas para poder dar respuestas a las necesidades particulares de sus estudiantes.

Los estudiantes con $\mathrm{NEE}$, pertenecientes al grupo experimental, lograron reconocer y representar el valor posicional solamente de unidades de primero, segundo y tercer orden, sin presentar ningún error y con apoyo de material concreto, gráfico o pedagógico, mientras que la mayoría (75 \%) de los estudiantes del grupo control, quienes cuentan con los mismos apoyos, aún no tienen conciencia del valor posicional de cada número dentro de una cifra, exceptuando un estudiante que se ubica en el nivel E.

Operaciones de suma y resta. En relación con la capacidad para formular, analizar y resolver situaciones matemáticas que involucren operaciones de sumas y restas, los estudiantes sin NEE del grupo experimental se encuentran ubicados entre los tres niveles conceptuales esperados (B, S y E); resalta que la mayoría (61.9 \%) logra utilizar las operaciones matemáticas a partir de la identificación de las palabras clave, y puede seleccionar la operación que le permite resolver correctamente la operación, respondiendo acertadamente la pregunta del problema. Los estudiantes del grupo control (75 \%) evidencian un retraso académico relacionado con la capacidad para formular, analizar y resolver situaciones matemáticas que involucren operaciones de sumas y restas.

Los estudiantes con NEE del grupo experimental han modificado su actividad de aprendizaje con el apoyo de materiales físicos (piedras, semillas, fichas, 
figuras geométricas), logrando adquirir la noción de cantidad y representar gráficamente la información relevante que se les plantea en un problema matemático, aunque sustituyen el isomorfismo por un esquema (líneas, círculos o cruces). En el grupo control se evidencia un avance más lento dado que no hacen nada, dicen que no saben o no pueden resolver el problema, en otros casos se limitan a jugar con el material concreto que se le provee o escriben letras, pseudografías o números arbitrariamente, es decir, que no guardan ninguna relación con los datos presentados en el planteamiento del problema.

\section{Solución de problemas}

Con respecto a la solución de problemas aritméticos de primer nivel, es decir, que involucran operaciones de suma y resta, los estudiantes sin NEE del grupo experimental intentan abandonar la representación pictórica como procedimiento para resolver problemas, pero al representarlos utilizando simbología matemática cometen errores; en otros casos solo utilizan la representación simbólica, pero con algunas fallas o utilizan una operación que no es adecuada con el enunciado del problema para hallar la solución del problema. Por el contrario, a los estudiantes del grupo control se les dificulta comprender el problema, e identificar los datos relevantes que este les ofrece; cuando se realiza la mediación algunos no responden a los interrogantes que se les plantean o simplemente manifiestan que no saben lo que tienen que hacer; otros logran relacionarlo con situaciones de la vida cotidiana, pero no pueden deducir el tipo de operación que deben realizar para solucionar correctamente el problema.

Con el apoyo de la representación gráfica y de algunas palabras clave, los estudiantes con algún tipo de NEE, pertenecientes al grupo experimental, logran hallar la solución de un problema matemático que requiera de una sola operación, mientras que los estudiantes del grupo control evidencian mayor dificultad para comprender el problema, representarlo y resolverlo independientemente, dado que generalmente responden que no saben y suspenden el ejercicio.

Es de resaltar que en cada prueba los estudiantes debían escribir el significado de cada concepto (suma, resta y problemas matemáticos) con el fin de evidenciar los saberes previos y poder determinar si a partir de la implementación del protocolo se logra que estos adquieran mayor comprensión y apropiación de los mismos. Al revisar las respuestas se encontró que 19 estudiantes del grupo experimental (el 63.3 \%) mejoraron el concepto de suma, ya que al definirlo utilizan palabras como agregar, aumentar; otros lo definen como una operación que da un número mayor y, en ocasiones, escriben ejemplos en forma de operación y lo resuelven en forma de problema que indica una suma.

Con respecto al concepto de resta se encuentra que 25 estudiantes (el 83.3\%) mejoraron en su definición al usar las palabras quitar, regalar o perder, 
las cuales son claves en la solución de estos problemas; además, se refieren a la diferencia como un número menor, lo que evidencia que asocian la operación con una disminución de cantidad.

Para la definición del concepto de problema matemático, inicialmente los estudiantes lo asociaban más con la convivencia (inconvenientes entre las personas); a partir de la experiencia, 13 estudiantes (el $43.33 \%$ ) modificaron su definición pasando a definirlo como una historia que se necesita resolver con la ayuda de las matemáticas; también hicieron el ejercicio de arriesgarse al escribir sus propios ejemplos de problemas matemáticos de tipo simple, lo que representa un elevado nivel metacognitivo, e incluso, utilizan palabras clave como "datos" para referirse a las cantidades que aparecen en el problema, lo cual no es muy usual en niños de este nivel.

\section{Conclusiones}

La investigación tuvo como objetivo general analizar si el uso de un protocolo de clase, en el grado segundo, como actividad orientadora de enseñanza, mejoraría la comprensión y la apropiación de los conceptos de suma y resta aplicados en la solución de problemas matemáticos. En concordancia con este fin, con la pregunta de investigación, con los referentes teóricos y con la propuesta metodológica que orientó dicha práctica, sumados al proceso formativo y reflexivo del equipo investigador, se expresan las siguientes conclusiones:

Esta investigación queda en un proceso de transición, al considerar que seis meses de aplicación, equivalentes a solo dos períodos académicos es un tiempo corto; sin embargo, se ha logrado apreciar que al implementar los pasos 1, 2 y 3 del protocolo de clase los estudiantes en general trasforman las prácticas en su actividad de aprendizaje, evidenciado en el aumento de la participación en los diálogos de clase, una actitud activa y de argumentación, que utiliza cada vez más un lenguaje propio de la asignatura de matemáticas.

Los pasos 1 y 2 abrieron la posibilidad de enseñar a través del diálogo, donde cada argumento, interrogante y aclaración pasaron a ser elementos articuladores entre los pre-saberes y los conceptos académicos que aporta la escuela Vigotsky (1983), ayudándoles en la adquisición de un metalenguaje, con el que fueron capaces de comprender cada concepto, y asociarlo con las dinámicas cotidianas, evidenciando la generalización del aprendizaje.

De manera más específica, en cuanto a los procesos de socialización y puesta en común de las diversas acciones ejecutadas durante el desarrollo de las actividades orientadoras de enseñanza propuestas desde los pasos 4, 5 y 6 del protocolo, los estudiantes han tenido la oportunidad de acceder a términos e información con los cuales no estaban familiarizados; también han desple- 
gado diversas estrategias como la aplicación consciente y menos mecánica de un algoritmo, la identificación de las palabras clave y los datos relevantes en un problema matemático que les posibilitan dar cuenta de cómo lo hicieron y generar discusiones tanto con los compañeros como con las docentes, resultado esperado en los pasos 7, 8 y 9 del protocolo.

En este ejercicio investigativo fue de vital importancia conocer las características cognitivas, comportamentales y académicas particulares de cada individuo, dado que esto pasó a ser un elemento importante para dimensionar las relaciones en el aula y el tipo de estrategias utilizadas como el protocolo de clase, el trabajo en equipo y las tutorías entre pares, sumados a estrategias dirigidas al proceso de construcción e interiorización de la norma por parte de los estudiantes, obteniendo como resultado, mayor autorregulación en su comportamiento, lo que posibilita, según Vigotsky (1983), la formación de conceptos por interacciones sociales y la verbalización del pensamiento.

El clima de libertad en el que se desarrolló esta experiencia permitió al equipo docente la reflexión constante sobre la acción de enseñar, los retos y compromisos adquiridos en el desempeño de la práctica pedagógica, y logró hacer de esta experiencia un espacio para diseñar estrategias, juegos y recursos que enriquecieran la acción didáctica, y transformaran su práctica de enseñanza y la mirada sobre la educación.

Las reflexiones anteriores permiten validar como un hallazgo favorable el haber encontrado una nueva estrategia para la organización de la enseñanza, ya que a través de las unidades didácticas acordes con cada uno de los pasos del protocolo como actividades orientadoras de enseñanza, y el registro sistemático del diario pedagógico, se pudieron replantear las prácticas pedagógicas para la formación de conceptos como la suma y la resta, aplicados en la solución de problemas matemáticos, respetando claro está, los diferentes niveles de conceptualización de los estudiantes, al adaptar dichas estrategias (lúdicas como de conceptualización) a los ritmos de aprendizaje y las necesidades educativas particulares o especiales de los estudiantes.

En investigaciones posteriores se espera extender en un tiempo más prolongado la estrategia del protocolo de clase como actividad orientadora de enseñanza, es decir, que corresponda a los cuatro períodos académicos de una institución educativa, y aplicarlo a un número mayor de estudiantes, así como ahondar en las causas de las dificultades que siguen presentando algunos estudiantes relacionadas con la apropiación del valor posicional, aspecto relevante para la formación y operacionalización de conceptos tanto de suma y resta como de multiplicación y división, propios de los problemas aritméticos de segundo nivel. 


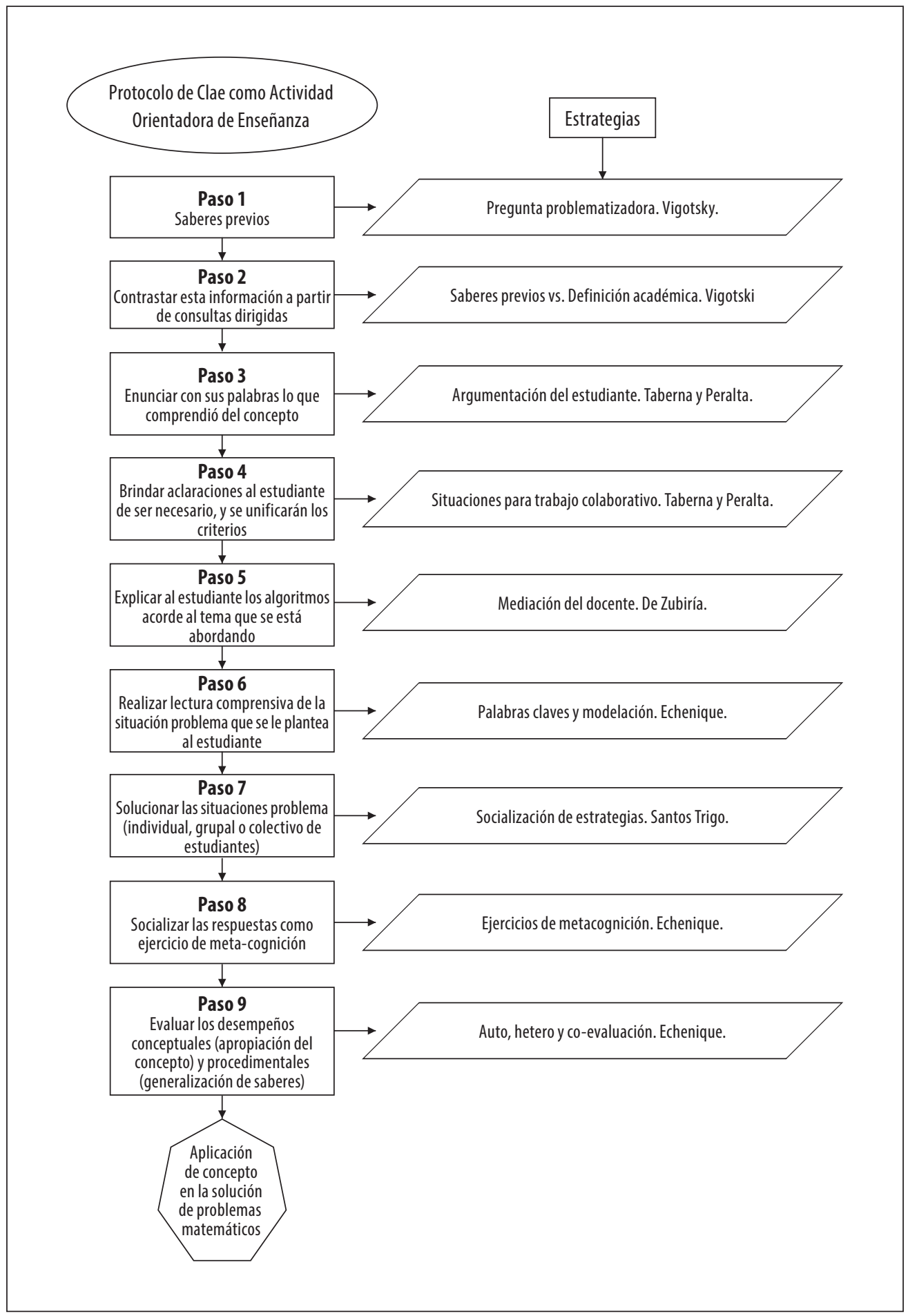




\section{Referentes bibliográficos}

Barcena et al. (2009). "100 propuestas para mejorar la competencia matemática”. Recuperado de http:// www.apega.org/attachments/article/856/propuestas_mejorar_competencias.pdf.

Blanco, B., y Blanco. L. (2009). "Contextos y estrategias en la resolución de problemas de primaria". Recuperado de http://funes.uniandes.edu.co/3513/1/Blanco2009ContextosNumeros71.pdf.

Castañeda, L. S. y Henao, J. I. (1995). La lectura en la universidad. Medellín: Universidad de Antioquia. Recuperado de https://aprendeenlinea.udea.edu.co/revistas/index.php/ikala/article/ viewFile/8543/7884.

De Zubiría, M., y De Zubiría, J. (1992). Biografía del pensamiento, estrategias para el desarrollo de la inteligencia. Santafé de Bogotá: Cooperativa Editorial Magisterio.

Delgado, M., Arrieta, X., y Campo H. (2012) Comparación de teorías relacionadas con la formación de conceptos científicos. En Multiciencias, Vol 12 (4), pp. 416-426.

Dewey, J. (1993). Compresión: Ideas y significado. En Cómo pensamos: nueva exposición de la relación entre pensamiento y proceso educativo (pp. 1221-132). Barcelona: Paidós.

Douglas, C., Bernaza, G., Corral, R., (2006). "Una propuesta didáctica para el aprendizaje de la física". Recuperado de http://rieoei.org/experiencias110.htm.

Echenique, I. (2006). "Matemáticas resolución de problemas primaria". Recuperado de http://dpto. educacion.navarra.es/publicaciones/pdf/matematicas.pdf.

Frontera, M. (1992). "Adquisición de los conceptos matemáticos básicos una perspectiva cognitiva" Tomo 1. Recuperado de http://biblioteca.ucm.es/tesis/19911996/S/5/S5001501.pdf.

Godino, J., Neto, T. (2013). "Actividades de iniciación a la investigación en educación matemática". Recuperado de https://ria.ua.pt/bitstream/10773/11825/1/jdgodino\%20UNO\%2063\%20Julio\%20 2013.pdf.

Hernández, H., (1997). "Vigotsky y la estructuración del conocimiento matemático. Experiencia Cubana". Recuperado de http://estadisticas.rimed.cu/interactividades.cubaeduca.cu/web/ soft _ eureka/module/profesor/visor _ articulos.php?ver=articulos\&cual=26\&num=15\&cat $=1 \&$ Tip $=$ sub\&idMod=15.

Hernández, H., (1997). "Vigotsky y la estructuración del conocimiento matemático. Experiencia Cubana". Recuperado de http://estadisticas.rimed.cu/interactividades.cubaeduca.cu/web/ soft_eureka/module/profesor/visor_articulos.php?ver=articulos\&cual=26\&num=15\&cat $=1 \&$ Tip $=$ sub\&idMod=15.

Jurado, F. (2004). "El dominio de los códigos de las ciencias y las matemáticas es el dominio de su lectura". Bogotá: Revista del Magisterio, N. ${ }^{\circ} 7$.

Martínez, J. (2011). Métodos de investigación cualitativa. Recuperado de http://www.cide.edu.co/ ojs/index.php/silogismo/article/viewFile/64/53.

Ministerio de Educación. Estándares básicos de competencias de matemáticas y lineamientos curriculares matemáticas. Recuperado de http://www.mineducacion.gov.co/1759/articles-116042_archivo_pdf2.pdf.

Negret, J (2008). Programa Cifras. Portafolio del educador. Colombia: Editorial Logoformas S. A. 
Rengifo, C. (2010). El protocolo académico. Recuperado de http://artemisa.unicauca.edu. $\mathrm{co} /$ gerardorengifo/Documentos/EduAmbDesarrolloRegional/El\%20protocolo2.pdf.

Sabina Taverna, S., y Peralta, O. (2009) Desarrollo conceptual: perspectivas actuales en adquisición temprana de conceptos. En Psykhe, Vol 18(1), pp. 49-59.doi:ISSN0717-0297

Sánchez, Y., González, F., Molina, O., Guil, M. (2009). "Guía para la elaboración de protocolos" Recuperado de http://www.index-f.com/lascasas/documentos/lc0565.pdf.

Santos, L. (1997). "Principios y métodos de la resolución de problemas en el aprendizaje de las matemáticas". Capítulo 6. Recuperado de http://fractus.uson.mx/geometria/UnidadIII/Lectura9b.pdf.

Solé, M. (2005). "El protocolo y la empresa". Recuperado de https://assets-libr.cantook.net/assets/ publications/4375/medias/excerpt.pdf.

Tamayo, M. (1999). Aprender a investigar. Módulo 2 La investigación. Recuperado de http://datateca. unad.edu.co/contenidos/209006/APRENDE__ A _ INVESTIGAR. _Mario _ Tamayo_y_ Tamayo.pdf.

Vigotsky, L (1983). Pensamiento y lenguaje. Buenos Aires: Editorial La Pléyade. 